\title{
ESTIMATIVA DO PERFIL DE VENTO E POTENCIAL EÓLICO OFFSHORE PARA COSTA DE CABO FRIO
}

Luiz Felipe Rodrigues do

\section{Carmo}

I.docarmo.meteoroufrj@gmail. com

Universidade Federal do Rio de Janeiro, Rio de Janeiro, Rio de Janeiro, Brasil.

\section{Ana Cristina Pinto de Almeida} Palmeira

anactn@gmail.com

Universidade Federal do Rio de Janeiro, Rio de Janeiro, Rio de Janeiro, Brasil.

Carlos Felipe de Jesus Lauriano Antonio

carlosfelipejla@gmail.com Universidade Federal do Rio de Janeiro, Rio de Janeiro, Rio de Janeiro, Brasil.

\section{RESUMO}

O homem é um grande consumidor de energia. Por conseguinte, estudar e melhorar os mecanismos de fornecimento de energia é de suma importância para a sociedade. Nessa temática, as energias renováveis vêm como um grande aliado ambiental em contraponto aos combustíveis fósseis. Alguns exemplos são: a energia solar, a eólica, a biomassa e a hidráulica. Nesse trabalho calculou-se a direção do vento, os perfis de velocidade do vento e potencial eólico para uma região próxima de Cabo Frio-RJ, utilizando os dados da boia de Cabo Frio (Nova) do Programa Nacional de Boias (PNBOIA) e os dados da reanálise do ERA5 de temperatura, velocidade, direção do vento e altura significativa da onda, para o período entre $01 / 08 / 2016$ e $31 / 07 / 2017$. Os resultados preliminares mostraram que a diferença absoluta média entre a velocidade do vento do PNBOIA e do ERA5 foi de aproximadamente $1 \mathrm{~m} / \mathrm{s}$. Além disso, através da rosa dos ventos, observa-se que não houve diferença significativa percentual entre as direções do vento do PNBOIA e do ERA 5. Concluiu-se que essa é uma região favorável para instalação de usinais eólicas.

Palavras-chave: perfil de vento; energia eólica; potencial eólico; ERA5; PNBOIA 


\section{INTRODUÇÃO}

Desde a existência humana, o homem vem buscando mecanismos que facilitem as suas atividades costumeiras. Dessa forma, ao dominar a técnica do fogo, ele melhorou sua qualidade de vida (CPFL Energia, 2017). Posteriormente, mais especificamente a partir da Revolução Industrial, os combustíveis fósseis, como o carvão mineral, apresentaram significativa mudança na vida da sociedade da época, sendo a principal fonte de energia.

Com o aumento da produção e exploração, houve um aumento significativo da quantidade de área explorada e das emissões antropogênicas e, como consequência, verifica-se desequilíbrio ambiental com a intensificação do efeito estu$\mathrm{fa}$, do aquecimento global e do desmatamento de biomas importantes, como o da Amazônia, do Pantanal, do Cerrado e da Mata Atlântica.

Nas últimas décadas, com o aprofundamento da discussão acerca de energias renováveis, foram desenvolvidas diferentes tecnologias como opções alternativas às tradicionais, a exemplo da energia solar, da eólica, da biomassa e da energia hidráulica. Dentre estas, a energia eólica é a que vem apresentando maior crescimento nos últimos anos.

Segundo Dutra (2008), o vento foi utilizado como recurso de auxílio para o desenvolvimento de diferentes tarefas cotidianas. Na agricultura, existiam os moinhos de vento, que consistiam em uma gaiola circular na qual animais ou homens caminhavam e moviam uma haste fincada em uma coluna vertical. As rodas d'água surgiram como aperfeiçoamento desse sistema, sendo sua força motriz o curso d'água.

Com os cata-ventos, a energia eólica foi empregada na moagem de grãos e bombeamento d'água. Na Europa, o uso dos cata-ventos iniciou-se com o retorno das Cruzadas, aproximadamente no século XIII, sendo por muito tempo bastante utilizado e documentado. O moinho de vento do tipo holandês, em especial, foi difundido por vários países, influenciando fortemente a economia agrícola do continente (Dutra, 2008).

Conforme se sabe, a energia eólica é aquela que provém do vento para produzir energia mecânica. Dessa forma, para que haja um bom aproveitamento energético e rentável, é necessário implementá-la em uma região com ventos constantes, com intensidade que possa variar entre moderada e muito forte. Além disso, preferencialmente, opta-se por regiões com menor cisalhamento na direção do vento, uma vez que, com a elevada variação na sua direção, faz-se necessário a implementação de aerogeradores com capacidade maior de rotação, de modo a captar a energia do vento em todas as direções, elevando ainda mais o custo (CEPEL, 2001).
Ohlenforst et al. (2018), através da Global Wind Report, mostraram a capacidade de energia eólica instalada por vários países, entre o período de 2015 a 2018. Nesse período, o Brasil quase dobrou a sua capacidade, apesar de ainda estar muito abaixo do ideal. Tendo em vista a Região Nordeste do Brasil, há um grande potencial de geração deste tipo energia, tanto onshore como offshore (Barcellos, 2014).

Segundo a Aneel (2020), o Nordeste é a região do Brasil com o maior potencial do território brasileiro, apresentando a maior quantidade de usinas eólicas. A região Sul também ganha destaque no painel nacional, possuindo um número considerável de parques em operação.

Apesar de possuir muitos parques eólicos, o Brasil ainda pode e precisa aumentar o número de usinas eólicas e também melhorar a sua gestão energética, uma vez que ainda há muitas áreas favoráveis a serem exploradas, e, portanto, começar a equilibrar a balança energética nacional. Também há uma grande área offshore na costa brasileira passível de ser explorada.

A energia eólica offshore vem ganhando evidência no mundo e se mostrando uma solução promissora para o futuro. Visto que a rugosidade do mar é menor que a do continente, o vento marítimo apresenta valores de intensidade superiores, o que garante ao campo offshore maior potencial eólico quando comparado ao onshore. Segundo Ohlerfost et al. (2018), há 4.496 instalações offshore no mundo, nenhuma delas no Brasil. Embora ainda não existam aerogeradores em operação em alto-mar, diversas empresas têm estudado a implementação de complexos eólicos pela costa brasileira.

Pelo exposto e tendo em vista a importância deste tema e do possível potencial de implementação de parques eólicos offshore, estudar a intensidade e direção do vento no oceano é muito importante. Contudo, analisar esses parâmetros apenas na superfície oceânica pode não ser eficiente, já que os aerogeradores normalmente são instalados em níveis superiores (por exemplo: 30, 60, 90 ou 100 metros). Além disso, quanto mais perto da camada superficial, maior é a influência da rugosidade na intensidade dos aerogeradores.

Tendo em vista essa problemática, torna-se importante estudar o perfil de vento e não apenas o vento em superfície, bem como calcular a energia e o potencial eólico para os níveis superiores. Sabendo disso, o objetivo desse trabalho é estudar este perfil de vento e calcular o perfil de potencial eólico em um ponto offshore localizado próximo da costa de Cabo Frio, região que corriqueiramente possui ventos em superfície de intensidade moderada à forte. 


\section{MÉTODOS E DADOS}

\section{Dados}

Para esse estudo, foram utilizados os dados de temperatura do ar, direção, intensidade do vento e altura significativa da onda da boia do Programa Nacional de Boias (PNBOIA) localizada em Cabo Frio, RJ, Brasil (Figura 1) e da reanálise do ERA5 do European Centre for Medium-Range Weather Forecasts (ECMWF), para o período entre 01/08/2016 e 31/07/2017.

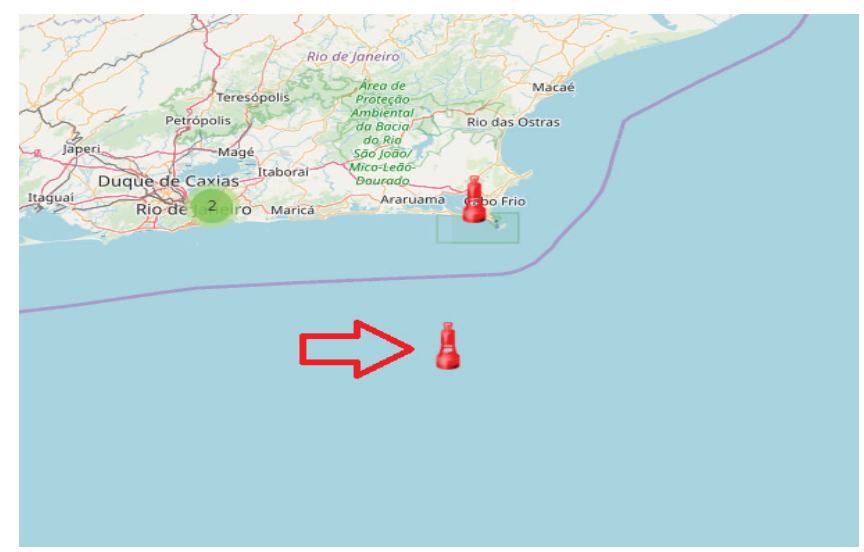

Figura 1. Localização das Boias do PNBOIA. Fonte: Marinha do Brasil

Dentro desse período, foi selecionado um período com ventos superiores a 30 nós (15 m/s), para realizar a comparação qualitativa do vento do ERA5 em relação aos dados da boia de Cabo Frio. Para tal, calculou-se a magnitude e direção do vento entre os dias 27/11/2016 e 09/12/2016. Quantitativamente, para o período entre 01/08/2016 e 31/07/2017 (1 ano), foram calculadas também métricas estatísticas comparativas entre a reanálise e a boia.

\section{Metodologia}

Para o cálculo do perfil de vento, foi utilizado o teorema de Pi-Buckingham (Kantha e Clayson, 2000) e a teoria da similaridade de Monin \& Obukhov (Monin e Obukhov, 1954; Wyngaard, 1973; Sorbjan, 1986; Stull, 1991). Integrando a equação 1 , obtém-se a equação 2 , de velocidade do vento para uma altura z.

$$
\begin{gathered}
\frac{k z}{u_{*}} \frac{\partial u}{\partial z}=\phi_{M} \\
u(z)=\frac{u_{*}}{k}\left[\ln \left(\frac{z}{z_{0}}\right)-\psi_{M}\left(\frac{z}{L}\right)\right]
\end{gathered}
$$

Onde $u$ é a velocidade do vento, $u$ é a velocidade de fricção, $k$ a constante de Von-Karman, $Z_{o}$ é a rugosidade e $L$ o comprimento de Monin-Obukhov.

Para estimativa da rugosidade, utilizou-se o método de Donelan (1990), obtido através de experimentos de campo, provindos de uma função direta da altura significativa de ondas descrita pela equação 3 .

$$
z_{0}=\xi\left(\frac{H_{s}}{4}\right)
$$

onde: $\mathrm{H}_{\mathrm{s}}$ é a altura significativa da onda e constante.

Existem diversos métodos de estimativa da potência mecânica extraída pelo aerogerador. Entretanto, nesse estudo foi utilizado o método de Pavinatto (2005), equação 4, que é um dos mais utilizados dentre os métodos mais recentes.

$$
P_{m}=\frac{1}{2} \rho A v_{w}^{3} c_{p}(\lambda, \beta)
$$

onde: $p$ é a densidade do ar, $A$ é a área varrida pelo rotor, $v_{w}$ é a velocidade incidente no rotor e $c_{p}$ é o coeficiente de potência do aerogerador.

O termo $c_{p}(\lambda, \beta)$ depende de características específicas da turbina eólica e ele é razão da função de razão de velocidades $(\lambda)$ e do ângulo de passo das pás da turbina eólica $(\beta)$. Como $c_{p}(\lambda, \beta)$ é bidimensional, algumas aproximações numéricas são realizadas. Dessa forma, nas equações 5 e 6 e na Figura 2 , encontram-se as aproximações numéricas utilizadas, bem como o gráfico característico de $c_{p}(\lambda, \beta)$, traçado a partir das equações 5 e 6 (Raiambal e Chellamuth, 2002; Pavinatto, 2005; Montezano, 2007).

$$
\begin{gathered}
c_{p}(\lambda, \beta)=0,5\left(\frac{98}{\lambda i}-0,4 \beta-5\right) e^{\frac{-16,5}{\lambda i}} \\
\lambda i=\frac{1}{\frac{1}{\lambda+0,089}-\frac{0,035}{\beta^{3}+1}}
\end{gathered}
$$

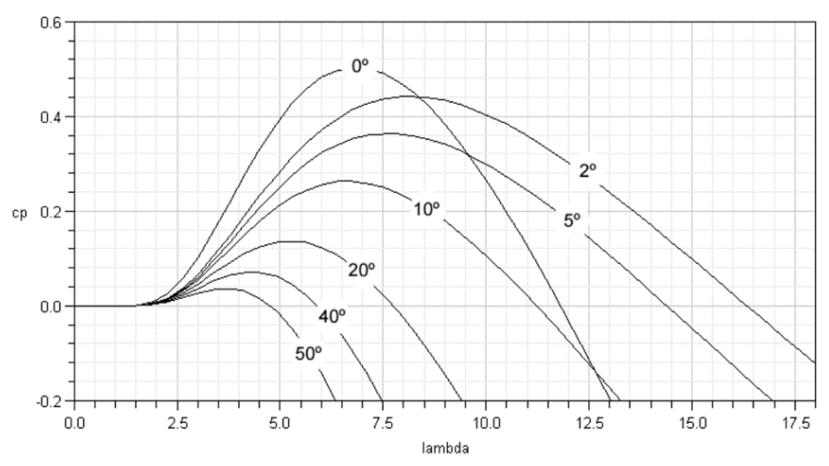

Figura 2. Gráfico de $c_{p}$ em função de lambda Fonte: Montezano, 2007 
Desse modo, para calcular a potência, foram escolhidos dois aerogeradores, sendo um de menor e um de maior capacidade: Modelo ELV-H2.7 - 500 W (Potência máxima de 1000W) e ELV-H20.8 - 100 kW (Potência máxima de $100000 \mathrm{~W})$, respectivamente.

\section{RESULTADOS}

Nas Figuras 3 e 4, são apresentadas, respectivamente, a diferença entre as medições de magnitude do vento do PNBOIA e do ERA5 e o gráfico de dispersão desses dados para Cabo Frio, no período de um ano (de 01/08/2016 a 31/07/2017). Conforme pode-se notar, a diferença média absoluta entre os dois é de $1,06 \mathrm{~m} / \mathrm{s}$ e sua correlação é alta $(R=0.87)$. $O$ gráfico de dispersão da Figura 4 indica que os valores de velocidade do vento monitorados pelo ERA5 são, em média, 10 metros menores que os valores do PNBOIA (respectivamente, $6,56 \mathrm{~m} / \mathrm{s}$ e $7,63 \mathrm{~m} / \mathrm{s}$ ). Ou seja, o ERA5 está subestimando a magnitude do vento em relação ao PNBOIA.

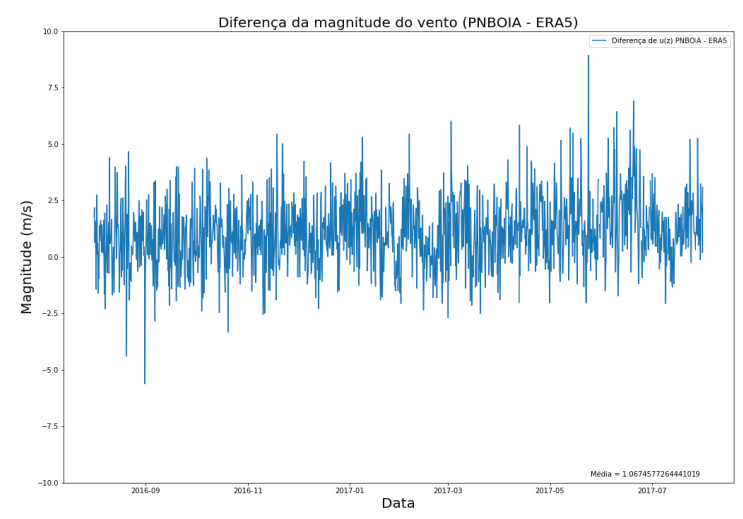

Figura 3. Diferença entre a magnitude do vento em 10 metros do PNBOIA e do ERA5

Nas Figuras 5, 6 e 7 encontram-se as variações da magnitude e da direção do vento tanto da boia de Cabo Frio, quanto do ERA5, durante o período de 27/11/2016 e 09/12/2016, e a variação da magnitude do vento de 0 a 100 metros para esse mesmo período. Nota-se que a magnitude do vento em níveis maiores do que 10 metros ultrapassou o valor de 15 $\mathrm{m} / \mathrm{s}$ em vários momentos, apresentando, por conseguinte, intensidade forte (Figura 7). Além disso, o erro médio absoluto da magnitude e da direção nesse período foi de aproximadamente $1,28 \mathrm{~m} / \mathrm{s}$ e $25^{\circ}$, respectivamente. Dessa forma, o erro da magnitude representou cerca de $13 \%$ do valor.

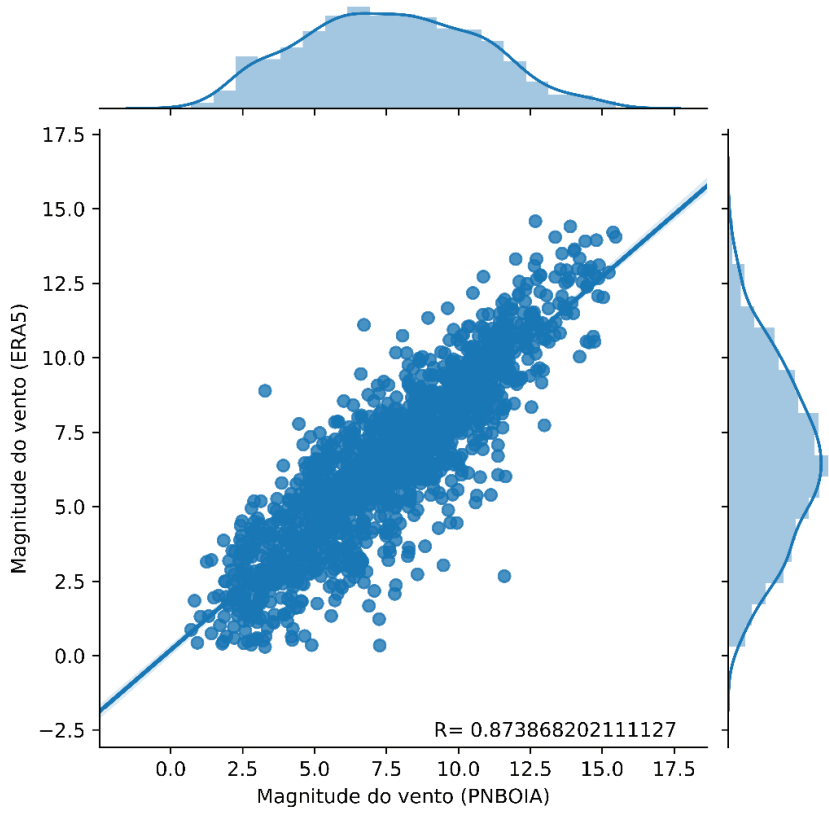

Figura 4. Dispersão da magnitude do vento em 10 metros (PNBOIA e ERA5)

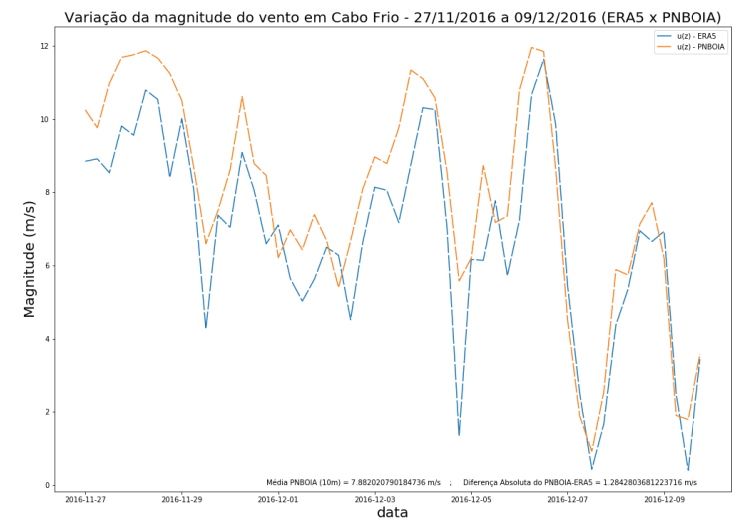

Figura 5. Variação da magnitude do vento (PNBOIA x ERA5)

Nas Figuras $8 \mathrm{a}$ e $8 \mathrm{~b}$ estão apresentadas a rosa dos ventos para o PNBOIA e ERA5 (localizada aproximadamente no ponto da boia de Cabo Frio) para o período de 1 ano. Percebe-se que as direções estão aproximadamente iguais, predominando a direção nordeste (NE), com aproximadamente $30 \%$ para o PNBOIA e $32 \%$ para o ERA5. Além disso, em ambas as fontes, mais de $60 \%$ dos ventos analisados encontraram-se entre as direções norte e leste.

A Figura 9 apresenta o perfil de vento anual médio $(0 \mathrm{a}$ 100 metros) do PNBOIA (vermelho) e do ERA5 (azul). Observa-se que, em razão da diferença de aproximadamente 1 $\mathrm{m} / \mathrm{s}$ entre a magnitude do vento a 10 metros, os valores dos níveis acima também se apresentaram diferentes e, portan- 
to, o ERA5 também subestima o vento em relação ao PNBOIA em níveis mais altos.

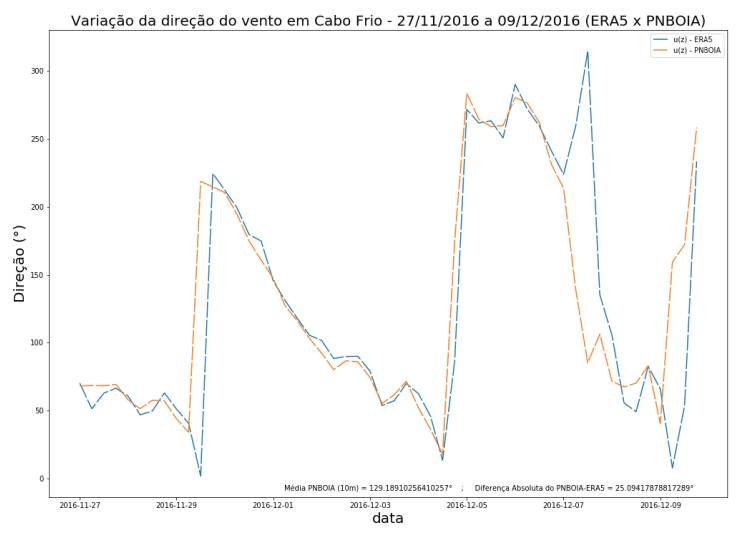

Figura 6. Variação da direção do vento (PNBOIA x ERA5)

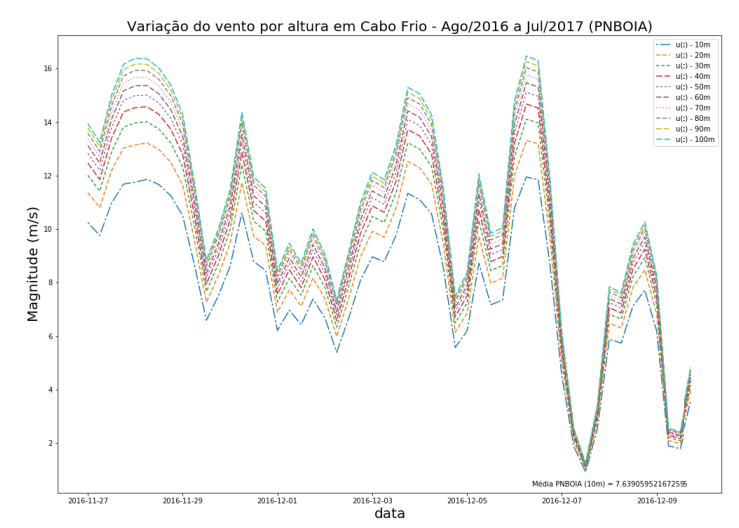

Figura 7. Variação da magnitude do vento de 0 a 100 metros (PNBOIA)

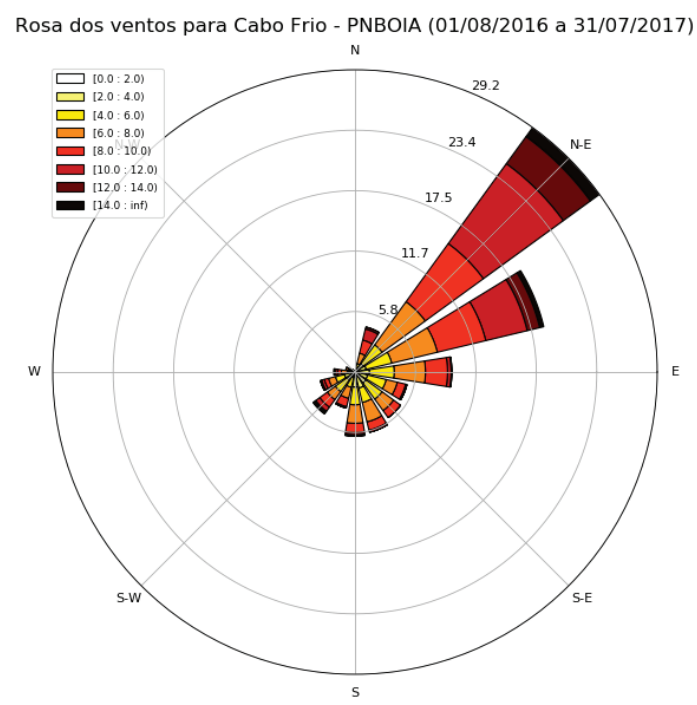

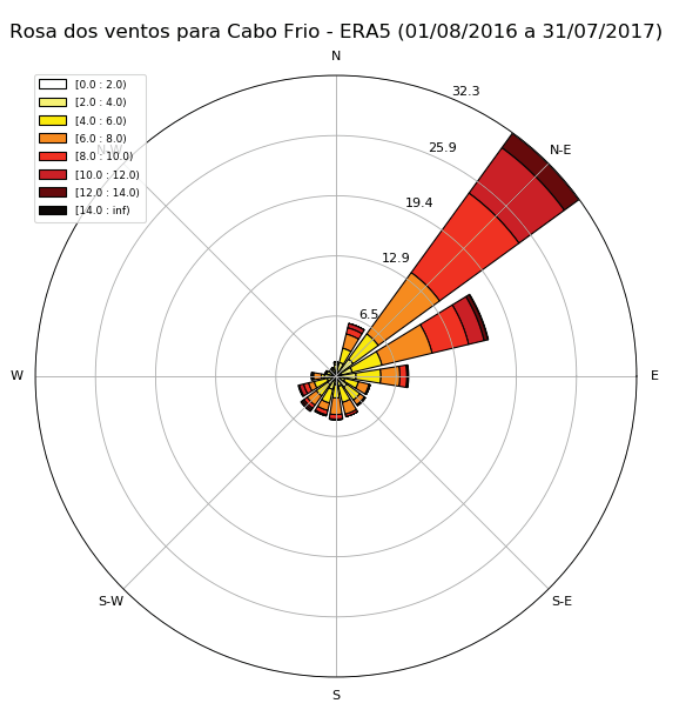

Figura 8. Rosa dos ventos para Cabo Frio do (a)PNBOIA e (b)ERA5.
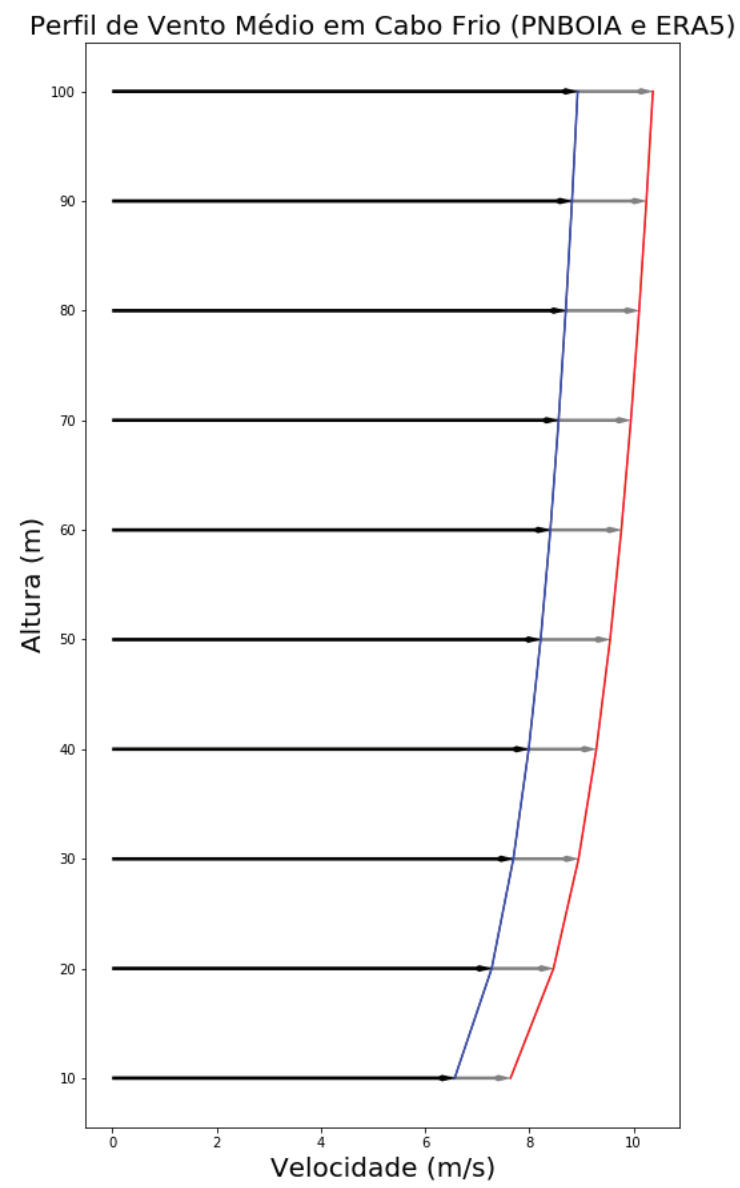

Figura 9. Perfil de vento para Cabo Frio [ERA5 (AZUL) + PNBOIA(VERMELHO)]

Com os perfis de vento já definidos, nas Figuras 10 e 11, pôde-se calcular os perfis de potencial eólico próximo à Cabo Frio do PNBOIA e ERA5 para os aerogeradores ELV-H2.7 
- 500 W e ELV-H20.8 - 100 kW. Devido ao fato da potência ser proporcional ao cubo da velocidade, ocorre proporcionalmente uma subestimativa da potência no ERA5. Portanto, no caso do aerogerador de menor potência (ELV-H2.7 $500 \mathrm{~W})$, a altura na qual é atingida a sua potência máxima seria de aproximadamente 18 metros para o PNBOIA e de 60 metros para o ERA5. Já para o ELV-H20.8 - 100 kW seria de 85 metros para o PNBOIA, enquanto para o ERA5 não se atinge o valor médio de $100 \mathrm{~kW}$ até o nível de 100 metros.

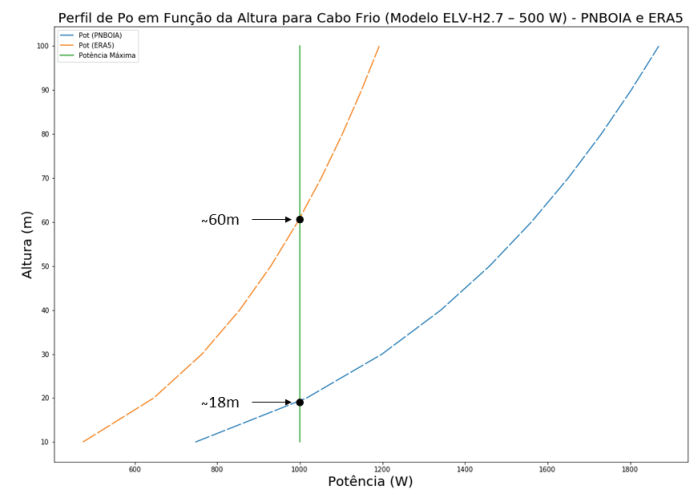

Figura 10. Perfil de potencial eólico próximo a Cabo Frio (ELV-H2.7 $-500 \mathrm{~W})$

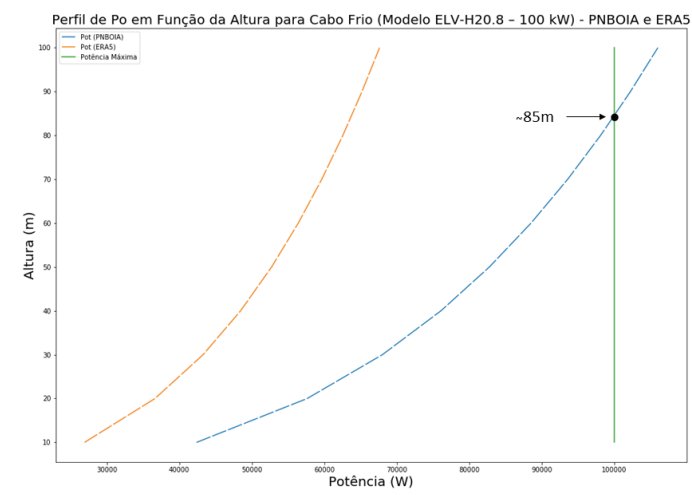

Figura 11. Perfil de potencial eólico próximo a Cabo Frio (ELV-H20.8-100 kW)

\section{CONCLUSÕES}

Os resultados mostraram que apesar do ERA5 ser uma reanálise, em termos direcionais das rosas dos ventos, a região de implantação do parque eólico, assim como da direção de instalação do aerogerador, seriam similares segundo o ERA5 e o PNBOIA. Entretanto, quando se olha do ponto de vista da magnitude, a subestimativa do ERA5 poderia implicar na determinação da altura de instalação do aerogerador.
Isso é um fator importante, uma vez que, sobre o oceano, a logística para instalação e operação dessas estruturas é muito mais complexa e cara.

Pode-se constatar, de acordo com os dados estudados, que a região de Cabo Frio, RJ parece ser região propícia para instauração desse tipo de malha energética, posto que a direção do vento não sofre variação tão grande e a sua magnitude é elevada, atingindo a potência máxima dos aerogeradores simulados. Entretanto, apesar dos resultados promissores, é necessário que outros estudos sejam realizados com um conjunto maior de dados, com novas metodologias de estimativa dos perfis e com um maior número de tipos de aerogeradores, para que se aumente ainda mais o conhecimento da região e das ferramentas utilizadas.

Um outro aspecto relevante é a interferência danosa da energia eólica offshore para o meio ambiente. Não se pode esquecer que é necessário um maior equilíbrio entre as malhas energéticas nacionais e a diminuição emergencial de combustíveis fósseis. Para tal, a energia eólica emerge como um aliado.

O instrumento capaz de resolver esse imbróglio e meIhorar a gestão de recursos ambientais é o licenciamento ambiental, pois, durante o pedido de licença prévia de instalação e de operação, cabe ao órgão ambiental (no caso desse tipo de licenciamento, o Instituto Brasileiro do Meio Ambiente e dos Recursos Naturais Renováveis - Ibama), determinar se o princípio da sustentabilidade (dentre outros) será preservado e se haverá benefícios para a sociedade.

\section{REFERÊNCIAS}

Agência Nacional de Energia Elétrica (ANEEL). 2020. Localização dos Parques Eólicos do Brasil. https://sigel.aneel.gov.br/ portal/home/webmap/viewer.html?useExisting=1

Barcellos, S.N. 2014. Avaliação de potencial eólico para aerogeradores de pequeno porte - Região de Estudo: Espírito Santo. Projeto de Graduação, Departamento de Engenharia Mecânica, Universidade Federal do Espírito Santo, Espírito Santo, Brasil.

Centro de Pesquisas de Energia Elétrica - CEPEL. 2001. Atlas do Potencial Eólico Brasileiro. Rio de Janeiro: CEPEL. http:// www.cresesb.cepel.br/publicacoes/download/atlas_eolico/ Atlas\%20do\%20Potencial\%20Eolico\%20Brasileiro.pdf

CPFL Energia. 2017. História da energia elétrica. https://www. cpfl.com.br/energias-sustentaveis/eficiencia-energetica/uso-consciente/historia-da-energia/Paginas/default.aspx

Donelan, M.A. 1990. Air-Sea Interaction, from The Sea: Ocean Engineering Science, Vol. 9 (two volume set), John Wiley \& Sons. 
Dutra, R. (Org.). 2008. Energia Eólica: Princípios e Tecnologias. Rio de Janeiro: Centro de Referência de Energia Solar e Eólica Sérgio de Salvo Brito. http://www.cresesb.cepel.br/download/tutorial/tutorial_eolica_2008_e-book.pdf

Kantha, L.H., Clayson, C.A. 2000. Small Scale Processes in Geophysical Fluid Flows, Academic Press, San Diego.

Monin, A.S.; Obukhov, A.M. 1954. Basic Laws of turbulent mixing in the atmosphere near the ground. Tr. Akad. Nauk. SSSR Geophiz. Inst. 24, 163-187.

Montezano, B.E.M. 2007. Modelo Dinâmico de Visualização de um Aerogerador com Velocidade de Rotação Variável e Controle de Passo em VRML. Projeto de Graduação, Departamento de Engenharia Elétrica, UFRJ, Rio de Janeiro, Brasil.

Ohlenforst, K., Sawyer, S., Dutton, A., Backwell, B., Fiestas, R., Lee, J., Qiao, L., Zhao, F., Balachandran, N. 2018. Global Wind Report. Global Wind Energy Council.
Pavinatto, E. F. 2005. Ferramenta para auxílio à análise de viabilidade técnica da conexão de parques eólicos à rede elétrica. Dissertação de Mestrado, Programa de Engenharia Elétrica, COPPE/UFRJ, Rio de Janeiro, Brasil.

Raiambal, K., Chellamuth, C. 2002. Modeling and Simulation of Grid Connected Wind Eletric Generating System, In: Proceedings of IEEE TENCON’02, pp. 1847-1852.

Sorbjan, Z. 1986. On Similarity in the atmospheric boundary layer. Bound. Layer Meteor. 43, 377-397.

Stull, R.B. 1991. An Introduction to Boundary Layer Meteorology. Dordrecht: Kluwer Academic Publishers.

Wyngaard, J. C. 1973. On Surface Layer Turbulence. Workshop on Micrometeorology, D.A. Haugen. American Meteorological Society, Boston, Mass, USA, pp. 101-148.

Recebido: 13 fev. 2020

Aprovado: 06 mar. 2020

DOI: 10.20985/1980-5160.2020.v15n1.1614

Como citar: Carmo, L.F.R., Palmeira, A.C.P.A., Antonio, C.F.J.L. (2020), Estimativa do perfil de vento e potencial eólico offshore para costa de Cabo Frio, Revista S\&G 15, No. 1, 46-52. https://revistasg.emnuvens.com.br/sg/ article/view/1614 